\title{
A NOTE ON SCHRÖDINGER MAXIMAL OPERATORS WITH A COMPLEX PARAMETER
}

\author{
PER SJÖLIN and FERNANDO SORIA ${ }^{凶}$
}

(Received 23 April 2009; accepted 26 January 2010)

Communicated by A. M. Hassell

Abstract

Extending previous results of the first author, some new estimates are obtained for maximal operators of Schrödinger type with a complex parameter.

2000 Mathematics subject classification: primary 42B15; secondary 42B25.

Keywords and phrases: maximal function, Schrödinger equation, Sobolev spaces.

\section{Introduction}

For $f$ belonging to the Schwartz class $\mathcal{S}(\mathbb{R})$, we set

$$
S_{t} f(x)=\int_{\mathbb{R}} e^{i x \xi} e^{i t \xi^{2}} \widehat{f}(\xi) d \xi \quad \forall x \in \mathbb{R} .
$$

Here $t$ is a complex number such that $\operatorname{Im} t \geq 0$, and $\widehat{f}$ denotes the Fourier transform of the function $f$, defined by

$$
\widehat{f}(\xi)=\int_{\mathbb{R}} e^{-i x \xi} f(x) d x .
$$

If we set $U(x, t)=(2 \pi)^{-1} S_{t} f(x)$, where $x \in \mathbb{R}$ and $t \in \mathbb{R}$, then it follows that $U(x, 0)=f(x)$ for all $x$ and further that $U$ satisfies the Schrödinger equation $i \partial U / \partial t=\partial^{2} U / \partial x^{2}$. On the other hand, if we take $t=i u$, where $u>0$, then $U$ is, modulo a constant, the solution to the usual heat equation with initial value $f$ with respect to the 'time variable' $u$.

We define the maximal function $S^{*} f$ by

$$
S^{*} f(x)=\sup _{0<t<1}\left|S_{t} f(x)\right| \quad \forall x \in \mathbb{R}
$$

The second author was partially supported by grants MTM2007-60952 and PR2009-0084.

(C) 2010 Australian Mathematical Publishing Association Inc. 1446-7887/2010 \$16.00 
and also define Sobolev spaces $H_{s}$ for all real $s$ by setting

$$
H_{s}=\left\{f \in \mathcal{S}^{\prime}:\|f\|_{H_{S}}<\infty\right\},
$$

where

$$
\|f\|_{H_{s}}=\left(\int_{\mathbb{R}^{n}}\left(1+|\xi|^{2}\right)^{s}|\widehat{f}(\xi)|^{2} d \xi\right)^{1 / 2} .
$$

It is well known that the estimate

$$
\left\|S^{*} f\right\|_{2} \leq C\|f\|_{H_{S}}
$$

holds if $s>1 / 2$ and does not hold if $s<1 / 2$ (see [1]). Here $\left\|S^{*} f\right\|_{2}$ denotes the norm of $S^{*} f$ in the space $L^{2}(\mathbb{R})$, and $C$ denotes a constant that varies from place to place.

When $0<\gamma<\infty$ and $u>0$, we set

$$
P_{u} f(x)=S_{u+i u^{\gamma}} f(x)=\int_{\mathbb{R}} e^{i x \xi} e^{i u \xi^{2}} e^{-u^{\gamma} \xi^{2}} \widehat{f}(\xi) d \xi \quad \forall x \in \mathbb{R}
$$

and

$$
P^{*} f(x)=\sup _{0<u<1}\left|P_{u} f(x)\right| \quad \forall x \in \mathbb{R} .
$$

In Sjölin [3] the inequality

$$
\left\|P^{*} f\right\|_{2} \leq C\|f\|_{H_{s}}
$$

was studied for various values of $\gamma$ and the following results were obtained.

THEOREM A.

(i) When $0<\gamma \leq 1$, (1.1) holds if and only if $s \geq 0$.

(ii) When $\gamma=2$, (1.1) holds if and only if $s \geq 1 / 4$.

(iii) When $\gamma \geq 4$, if (1.1) holds then $s \geq 1 / 2-1 / \gamma$.

When $\gamma>0$, we denote by $E_{\gamma}$ the set of all $s$ such that (1.1) holds, and set

$$
s(\gamma)=\inf E_{\gamma} .
$$

It was proved in [3] that $s$ is a nondecreasing function on the interval $(0, \infty)$, and that $0 \leq s(\gamma) \leq 1 / 2$ when $0<\gamma<\infty$.

The results in Theorem A can be stated in the following way.

THEOREM B.

(i) When $0<\gamma \leq 1, s(\gamma)=0$.

(ii) $s(2)=1 / 4$.

(iii) When $\gamma>4,1 / 2-1 / \gamma \leq s(\gamma) \leq 1 / 2$ and hence

$$
\lim _{\gamma \rightarrow \infty} s(\gamma)=1 / 2
$$

We give here the following improvement of the above results. 
THEOREM 1.1. If $\gamma>1$ and $s>1 / 2-1 /(2 \gamma)$, then (1.1) holds.

The result in Theorem 1.1 is new when $1<\gamma<2$ and $\gamma>2$, and allows us to extend Theorem B in the following way.

THEOREM 1.2.

(i) When $0<\gamma \leq 1$, $s(\gamma)=0$.

(ii) When $1<\gamma<2$, $0 \leq s(\gamma) \leq 1 / 2-1 /(2 \gamma)$.

(iii) $s(2)=1 / 4$.

(iv) When $2<\gamma \leq 4,1 / 4 \leq s(\gamma) \leq 1 / 2-1 /(2 \gamma)$.

(v) When $\gamma>4,1 / 2-1 / \gamma \leq s(\gamma) \leq 1 / 2-1 /(2 \gamma)$.

\section{Proof of the theorems}

For the proof of the above results we shall use the following lemmas.

Lemma 2.1. Assume that $a>1,1 / 2 \leq s<1$ and $\mu \in \mathcal{C}_{0}^{\infty}(\mathbb{R})$. Then

$$
\left.\left|\int_{\mathbb{R}} e^{i x \xi+i t|\xi|^{a}}\right| \xi\right|^{-s} \mu(\xi / N) d \xi \mid \leq C \frac{1}{|x|^{1-s}} \quad \forall x \in \mathbb{R} \backslash\{0\},
$$

when $t \in \mathbb{R}$ and $N=1,2,3, \ldots$. Here the constant $C$ may depend on $s$ and a but not on $x, t$ or $N$.

A proof of Lemma 2.1 can be found in [2].

LEMMA 2.2. Assume that $1 / 2 \leq \alpha<1$ and $0<d_{1}, d_{2}<1$, and also that $\mu \in \mathcal{C}_{0}^{\infty}(\mathbb{R})$ is even and real-valued. Then

$$
\begin{aligned}
& \left|\int_{\mathbb{R}} \exp \left(i\left(d_{1}-d_{2}\right) \xi^{2}-i x \xi\right)\left(1+\xi^{2}\right)^{-\alpha / 2} \exp \left(-\left(d_{1}^{2}+d_{2}^{2}\right) \xi^{2}\right) \mu(\xi / N) d \xi\right| \\
& \quad \leq K(x) \quad \forall x \in \mathbb{R}
\end{aligned}
$$

when $N=1,2,3, \ldots$, where $K \in L^{1}(\mathbb{R})$. Here $K$ is independent of $d_{1}, d_{2}$ and $N$.

Lemma 2.2 is proved in [3].

We also need two new lemmas.

LEMMA 2.3. Assume that $1<\gamma<2,(\gamma-1) / \gamma<\alpha<1 / 2,0<d_{1}, d_{2}<1$, and $\mu$ is as in Lemma 2.2. Then

$$
\begin{aligned}
& \left|\int_{\mathbb{R}} \exp \left(i\left(d_{1}-d_{2}\right) \xi^{2}-i x \xi\right)\left(1+\xi^{2}\right)^{-\alpha / 2} \exp \left(-\left(d_{1}^{\gamma}+d_{2}^{\gamma}\right) \xi^{2}\right) \mu(\xi / N) d \xi\right| \\
& \quad \leq K(x) \quad \forall x \in \mathbb{R}
\end{aligned}
$$

when $N=1,2,3, \ldots$, where $K \in L^{1}(\mathbb{R})$. Here $K$ is independent of $d_{1}, d_{2}$ and $N$. 
LEMMA 2.4. Assume that $\gamma>2,(\gamma-1) / \gamma<\alpha<1,0<d_{1}, d_{2}<1$, and $\mu$ is as in Lemma 2.2. Then

$$
\begin{aligned}
& \left|\int_{\mathbb{R}} \exp \left(i\left(d_{1}-d_{2}\right) \xi^{2}-i x \xi\right)\left(1+\xi^{2}\right)^{-\alpha / 2} \exp \left(-\left(d_{1}^{\gamma}+d_{2}^{\gamma}\right) \xi^{2}\right) \mu(\xi / N) d \xi\right| \\
& \quad \leq K(x) \quad \forall x \in \mathbb{R}
\end{aligned}
$$

when $N=1,2,3, \ldots$, where $K \in L^{1}(\mathbb{R})$. Here $K$ is independent of $d_{1}, d_{2}$ and $N$.

We now give the proofs of Lemmas 2.4 and 2.3.

Proof of Lemma 2.4. Let $C_{0}$ denote a large constant. Since $1 / 2<\alpha<1$, in the case where $|x| \leq C_{0}$ we can use the proof in [2] of Lemma 2.1 to conclude that the estimate in Lemma 2.4 holds when $K(x)=C|x|^{\alpha-1}$. To obtain this, we have to use the observation (see [3]) that if $h(\xi)=h_{\epsilon}(\xi)=e^{-\epsilon \xi^{2}}$ where $0<\epsilon<2$, then

$$
\left|h^{\prime}(\xi)\right| \leq C \frac{1}{\xi} \quad \forall \xi \in[1 / 2, \infty),
$$

where $C$ is independent of $\epsilon$.

We now consider the case where $|x|>C_{0}$. To that end, we shall modify the proof in [3] of our Lemma 2.2.

We may assume that $d_{2}<d_{1}$ and set $d=d_{1}-d_{2}$ and $\epsilon=d_{1}^{\gamma}+d_{2}^{\gamma}$, so that $0<d<1$ and $0<\epsilon<2$. Also set $\rho=|x| /(2 d)$ and

$$
\psi(\xi)=\left(1+\xi^{2}\right)^{-\alpha / 2} e^{-\epsilon \xi^{2}} \mu(\xi / N) \quad \forall \xi \in \mathbb{R} .
$$

Choose an even function $\varphi_{0} \in \mathcal{C}^{\infty}$ such that $\varphi_{0}(\xi)=1$ if $|\xi| \leq 1 / 2$ and $\varphi(\xi)=0$ if $|\xi| \geq 1$. Set $\psi_{0}=\psi \varphi_{0}$, so that supp $\psi_{0} \subset[-1,1]$. Then, for a large constant $K_{1}$, choose $\varphi_{2} \in \mathcal{C}_{0}^{\infty}$ so that $\operatorname{supp} \varphi_{2} \subset\left[\rho / 4,2 K_{1} \rho\right]$ and $\varphi_{2}(\xi)=1$ if $\rho / 2 \leq \xi \leq K_{1} \rho$. We may also assume that $\left|\varphi_{2}^{\prime}(\xi)\right| \leq C \xi^{-1}$ and $\left|\varphi_{2}^{\prime \prime}(\xi)\right| \leq C \xi^{-2}$ if $\xi>0$. We also set $\varphi_{3}=\left(1-\varphi_{2}\right) \chi_{\left[K_{1} \rho, \infty\right)}$ and $\varphi_{1}=\left(1-\varphi_{2}-\varphi_{0}\right) \chi_{[0, \rho / 2]}$.

Having defined the cutoff functions $\varphi_{j}$, where $j=0,1,2,3$, it is clear that it is sufficient to estimate the integrals

$$
\mathcal{J}_{j}=\int e^{i F} \psi_{j} d \xi
$$

where $F(\xi)=d \xi^{2}-x \xi$ and $\psi_{j}(\xi)=\psi(\xi) \varphi_{j}(\xi)$. (A similar argument works for the functions $\psi(\xi) \varphi_{j}(-\xi)$.) A double integration by parts easily shows the estimate $\left|\mathcal{J}_{0}\right| \leq C /|x|^{2}$ (see [3]). Now observe that when $j=1,2,3$ and $\xi>1 / 2$, the pointwise estimates

$$
\begin{aligned}
& \left|\psi_{j}(\xi)\right| \leq C \frac{1}{\left(1+\xi^{2}\right)^{\alpha / 2}}, \\
& \left|\psi_{j}^{\prime}(\xi)\right| \leq C \frac{1}{\left(1+\xi^{2}\right)^{\alpha / 2} \xi},
\end{aligned}
$$


and

$$
\left|\psi_{j}^{\prime \prime}(\xi)\right| \leq C \frac{1}{\left(1+\xi^{2}\right)^{\alpha / 2} \xi^{2}}
$$

hold. Using the same arguments as in [3], we obtain the estimate $\mathcal{O}\left(|x|^{-2}\right)$ for $\mathcal{J}_{1}$ and $\mathcal{J}_{3}$.

To estimate $\mathcal{J}_{2}$, we use van der Corput's lemma and deduce that

$$
\begin{aligned}
\left|\mathcal{J}_{2}\right| & \leq C d^{-1 / 2} \rho^{-\alpha} \exp \left(-c \epsilon \rho^{2}\right) \\
& \leq C d^{-1 / 2}\left(\frac{|x|}{d}\right)^{-\alpha} \exp \left(-c\left(d_{1}^{\gamma}+d_{2}^{\gamma}\right)|x|^{2} / d^{2}\right) \\
& \leq C d^{\alpha-1 / 2}|x|^{-\alpha} \exp \left(-c\left(d_{1}+d_{2}\right)^{\gamma}|x|^{2} / d^{2}\right) \\
& \leq C d^{\alpha-1 / 2}|x|^{-\alpha} \exp \left(-c d^{\gamma-2}|x|^{2}\right),
\end{aligned}
$$

where we have used the fact that $d_{1}+d_{2} \geq d$. Here $c$ denotes possibly different positive constants.

We now invoke the inequality

$$
e^{-y} \leq C_{\beta} y^{-\beta}
$$

which holds whenever $y>0$ and $\beta>0$, to deduce that

$$
\begin{aligned}
\left|\mathcal{J}_{2}\right| & \leq C d^{\alpha-1 / 2}|x|^{-\alpha} \frac{1}{d^{(\gamma-2) \beta}|x|^{2 \beta}} \\
& =C \frac{d^{\alpha-1 / 2}}{d^{\beta(\gamma-2)}} \frac{1}{|x|^{\alpha+2 \beta}} .
\end{aligned}
$$

We now choose $\beta$ so that $\beta(\gamma-2)=\alpha-1 / 2$, that is,

$$
\beta=\frac{\alpha-1 / 2}{\gamma-2} \text {. }
$$

Since $\gamma>2$ and $1 / 2<\alpha<1$, it is clear that $\beta$ is positive. We obtain the inequality

$$
\left|\mathcal{J}_{2}\right| \leq C \frac{1}{|x|^{\alpha+2 \beta}} .
$$

Finally, using our assumption that $\alpha>(\gamma-1) / \gamma$, we get

$$
\alpha+2 \beta=\frac{\alpha \gamma-1}{\gamma-2}>\frac{\gamma-1-1}{\gamma-2}=1 \text {. }
$$

Hence the function $|x|^{-\alpha-2 \beta}$ is integrable when $|x|>C_{0}$ and the proof of Lemma 2.4 is complete.

Proof of Lemma 2.3. As before, we let $C_{0}$ denote a large constant. We first study the case where $|x|>C_{0}$. With the same notation as in the previous proof and the arguments in [3], the estimates for $\mathcal{J}_{0}, \mathcal{J}_{1}$ and $\mathcal{J}_{3}$ follow easily. (Observe that the condition $\alpha \geq 1 / 2$ was not used for these estimates.) 
To estimate $\mathcal{J}_{2}$ we use van der Corput's lemma again and deduce that

$$
\begin{aligned}
\left|\mathcal{J}_{2}\right| & \leq C d^{-1 / 2} \rho^{-\alpha} e^{-c \epsilon \rho^{2}} \\
& \leq C d^{\alpha-1 / 2}|x|^{-\alpha} e^{-c d^{\gamma-2}|x|^{2}} .
\end{aligned}
$$

Using inequality (2.1), we then obtain

$$
\begin{aligned}
\left|\mathcal{J}_{2}\right| & \leq C d^{\alpha-1 / 2}|x|^{-\alpha} \frac{1}{d^{(\gamma-2) \beta}|x|^{2 \beta}} \\
& =C \frac{d^{\beta(2-\gamma)}}{d^{1 / 2-\alpha}} \frac{1}{|x|^{\alpha+2 \beta}} .
\end{aligned}
$$

Here $2-\gamma>0$ and, therefore, $1 / 2-\alpha>0$. Choosing $\beta$ large, we conclude that

$$
\left|\mathcal{J}_{2}\right| \leq C \frac{1}{|x|^{\alpha+2 \beta}} \leq C \frac{1}{|x|^{2}} .
$$

This completes the proof in the case where $|x|>C_{0}$. It remains to study the case where $|x| \leq C_{0}$. To do so, we modify the arguments given in the proof of Lemma 2.1 (see [2]). Since $\alpha<1 / 2$, we need a different argument to estimate

$$
\int_{I_{2}} e^{i F} \psi d \xi
$$

where, for some constants $c_{1}$ small and $C_{1}$ large, $I_{2}$ denotes the interval

$$
I_{2}=\left\{\xi \geq \frac{1}{|x|}: c_{1} \frac{|x|}{d} \leq \xi \leq C_{1} \frac{|x|}{d}\right\}
$$

Also,

$$
\begin{gathered}
F(\xi)=-x \xi+d \xi^{2}, \\
\psi(\xi)=\left(1+\xi^{2}\right)^{-\alpha / 2} e^{-\epsilon \xi^{2}} \mu(\xi / N) \quad \forall \xi \in \mathbb{R},
\end{gathered}
$$

and $d=d_{1}-d_{2}, \epsilon=d_{1}^{\gamma}+d_{2}^{\gamma}$. The rest of the proof is unchanged.

Set $\rho=|x| /(2 d)$ as before. Arguing as in the proof of Lemma 2.2, we deduce that

$$
|\psi| \leq C \rho^{-\alpha} e^{-c \epsilon \rho^{2}}
$$

on $I_{2}$, and

$$
\int_{I_{2}}\left|\psi^{\prime}\right| d \xi \leq C \rho^{-\alpha} e^{-c \epsilon \rho^{2}} .
$$

An application of van der Corput's lemma then yields

$$
\left|\int_{I_{2}} e^{i F} \psi d \xi\right| \leq C d^{-1 / 2} \rho^{-\alpha} e^{-c \epsilon \rho^{2}} .
$$


Arguing as in the previous case, we obtain the estimate

$$
\left|\int_{I_{2}} e^{i F} \psi d \xi\right| \leq C \frac{d^{\beta(2-\gamma)}}{d^{1 / 2-\alpha}} \frac{1}{|x|^{\alpha+2 \beta}} .
$$

Choosing

$$
\beta=\frac{1 / 2-\alpha}{2-\gamma}
$$

it follows that

$$
\left|\int_{I_{2}} e^{i F} \psi d \xi\right| \leq C \frac{1}{|x|^{\alpha+2 \beta}}
$$

and using our assumption that $\alpha>(\gamma-1) / \gamma$, we get

$$
\alpha+2 \beta=\frac{1-\alpha \gamma}{2-\gamma}<\frac{1-(\gamma-1)}{2-\gamma}=1
$$

Hence, the function $x \mapsto|x|^{-\alpha-2 \beta}$ is integrable in the interval $|x| \leq C_{0}$ and the proof of Lemma 2.3 is complete.

Finally, we give the proof of Theorem 1.1.

Proof of Theorem 1.1. As in [3, Theorem 1], we only need to prove that

$$
\left\|T_{N}^{*} h\right\|_{2} \leq C\|h\|_{2},
$$

when $N=1,2,3, \ldots$, where the operators $T_{N}^{*}$ are defined by

$$
T_{N}^{*} h(\xi)=\rho_{N}(\xi)\left(1+\xi^{2}\right)^{-s / 2} \int_{\mathbb{R}} e^{-i x \xi} e^{-i u(x) \xi^{2}} e^{-(u(x))^{\gamma} \xi^{2}} \chi_{N}(x) h(x) d x .
$$

Here $\chi_{N}(x)=\chi(x / N), \rho_{N}(\xi)=\rho(\xi / N)$ and $\chi, \rho \in \mathcal{C}_{0}^{\infty}(\mathbb{R})$ are such that

$$
\chi(x)=\rho(x)= \begin{cases}1 & \text { when }|x| \leq 1 \\ 0 & \text { when }|x| \geq 2\end{cases}
$$

and both $\chi$ and $\rho$ are even and real-valued. Further, $u$ is a measurable function on $\mathbb{R}$ such that $0<u(x)<1$. Invoking Lemmas 2.3 or 2.4 , we then have

$$
\begin{aligned}
\left\|T_{N}^{*} h\right\|_{2}^{2}= & \int T_{N}^{*} h(\xi) \overline{T_{N}^{*} h(\xi)} d \xi \\
= & \int \rho_{N}(\xi)^{2}\left(1+\xi^{2}\right)^{-s}\left(\int_{\mathbb{R}} e^{-i x \xi} e^{-i u(x) \xi^{2}} e^{-(u(x))^{\gamma} \xi^{2}} \chi_{N}(x) h(x) d x\right) \\
& \times\left(\int_{\mathbb{R}} e^{i y \xi} e^{i u(y) \xi^{2}} e^{-(u(y))^{\gamma} \xi^{2}} \chi_{N}(y) \overline{h(y)} d y\right) d \xi
\end{aligned}
$$


Here, when $1<\gamma<2$ we have assumed, as we may, that $1 / 2-1 /(2 \gamma)<s<1 / 4$. If $\alpha=2 s$ and $1<\gamma<2$, then

$$
1-1 / \gamma<\alpha<1 / 2
$$

Also, if $\alpha=2 s$ and $\gamma>2$, then we will assume that $1 / 2-1 /(2 \gamma)<s<1 / 2$, so that

$$
1-1 / \gamma<\alpha<1
$$

Hence, setting $\mu=\rho^{2}$ and applying Lemmas 2.3 and 2.4,

$$
\begin{aligned}
\left\|T_{N}^{*} h\right\|_{2}^{2}=\iint & \left(\int\left(1+\xi^{2}\right)^{-s} \exp (i(y-x) \xi) \exp \left(i(u(y)-u(x)) \xi^{2}\right)\right. \\
& \left.\times \exp \left(-\left((u(y))^{\gamma}+(u(x))^{\gamma}\right) \xi^{2}\right) \mu(\xi / N) d \xi\right) \\
& \times \chi_{N}(x) \chi_{N}(y) h(x) \overline{h(y)} d x d y \\
\leq C & \iint K(x-y)|h(x)||h(y)| d x d y \leq C\|h\|_{2}^{2} .
\end{aligned}
$$

Hence (2.2) is proved, and the proof of Theorem 1.1 is complete.

\section{References}

[1] P. Sjölin, 'Global maximal estimates for solutions to the Schrödinger equation', Studia Math. 110 (1994), 105-114.

[2] P. Sjölin, 'Maximal estimates for solutions to the nonelliptic Schrödinger equation', Bull. Lond. Math. Soc. 39 (2007), 404-412.

[3] P. Sjölin, 'Maximal operators of Schrödinger type with a complex parameter', Math. Scand. 105 (2009), 121-133.

PER SJÖLIN, Department of Mathematics, Royal Institute of Technology,

S-100 44 Stockholm, Sweden

e-mail: pers@math.kth.se

FERNANDO SORIA, Departamento de Matemáticas,

Universidad Autónoma de Madrid and Instituto de Ciencias Matemáticas, ICMAT,

E-28049 Madrid, Spain

e-mail: fernando.soria@uam.es 